\title{
SUMS OF SQUARES OF POLYNOMIALS WITH RATIONAL COEFFICIENTS
}

\author{
CLAUS SCHEIDERER
}

\begin{abstract}
We construct families of explicit polynomials $f$ over $\mathbb{Q}$ that are sums of squares of polynomials over $\mathbb{R}$, but not over $\mathbb{Q}$. Whether or not such examples exist was an open question originally raised by Sturmfels. We also study representations of $f$ as sums of squares of rational functions over $\mathbb{Q}$. In the case of ternary quartics, we prove that our counterexamples to Sturmfels' question are the only ones.
\end{abstract}

\section{INTRODUCTION}

Let $f\left(x_{1}, \ldots, x_{n}\right)$ be a polynomial with rational coefficients, and assume that $f$ is a sum of squares of polynomials with real coefficients. A few years ago, Sturmfels raised the question whether $f$ is necessarily a sum of squares of polynomials with rational coefficients. The main result of this paper gives a negative answer to this question.

The background for this question comes from semidefinite programming (see e.g. [16, [5], 10, 1]) and more specifically, from polynomial optimization. Lasserre's method of moment relaxation [9] gives, in principle, positivity certificates for real polynomials based on sums of squares decompositions. However, even if the initial data is exact, e.g. given by polynomials with rational coefficients, the algorithm produces floating point solutions, and therefore the output is not necessarily reliable. One would like to understand to what extent one can expect exact certificates, see for instance [12, 8. The question by Sturmfels addresses this issue in its most basic form.

From general reasons, it is clear that $f$ has a sum of squares representation over some real number field $K$. So far, it was known by work of Hillar [7 that the question has a positive answer when $K$ is totally real. Under this assumption, Hillar also gave a bound for the number of squares needed over $\mathbb{Q}$, in terms of the number needed over $K$ and of the degree of the Galois closure of $K$ over $\mathbb{Q}$. Quarez [1] later gave a different proof to the same result and improved Hillar's bound significantly. Both proofs are constructive. In Section 1 we revisit the result and show that it is essentially an immediate consequence of well-known properties of the trace form of $K / \mathbb{Q}$. Our argument is constructive as well. In addition it gives various new information, for instance that the bound found by Quarez holds in the non-Galois as well.

In Section 2 we present explicit counterexamples to the question by Sturmfels. Working with homogeneous polynomials (forms) we construct, for any integer $n \geq 2$ and any even number $d \geq 4$, a family of forms $f \in \mathbb{Q}\left[x_{0}, \ldots, x_{n}\right]$ of degree $d$ that are sums of two squares of forms over $\mathbb{R}$, but not sums of squares of forms

Date: September 10, 2018. 
over $\mathbb{Q}$ (Theorem 2.1). These forms $f$ are the $K / \mathbb{Q}$-norms of linear forms defined over suitable number fields $K$ of degree $d$. As a by-product, we show for any real number field $k$ that there is no analogue of Hilbert's theorem on nonnegative ternary quartics (the qualitative part): There always exists a nonnegative ternary quartic form with coefficients in $\mathbb{Q}$ that is not a sum of squares of forms over $k$ (Corollary 2.11).

Any nonnegative form $f \in \mathbb{Q}\left[x_{0}, \ldots, x_{n}\right]$ is a sum of squares of rational functions over $\mathbb{Q}$, according to Artin. In Section 3 we study such representations for the family of counterexamples constructed in Section 2 . If $f$ is such a form with $\operatorname{deg}(f)=d$, we prove (Theorem 3.3) that there always exists a nonzero form $h$ over $\mathbb{Q}$ of degree $d-2$, but not of any smaller degree, for which $f h$ is a sum of squares over $\mathbb{Q}$. In fact, we explicitly construct all such forms $h$ (Proposition [3.4). For $d=4$, this yields in particular an explicit representation of $f$ as a sum of squares of rational functions à la Artin.

In Section 4 we prove a partial converse to the construction from Section 2, In the case $(n, d)=(2,4)$ of ternary quartics, we show that every counterexample to Sturmfels' question arises from our construction (Theorem 4.1). The proof makes use of a canonical linear subspace $U_{f} \subseteq \mathbb{R}\left[x_{0}, \ldots, x_{n}\right]$ associated with any sum of squares $f \in \mathbb{R}\left[x_{0}, \ldots, x_{n}\right]$. We call $U_{f}$ the characteristic subspace associated with $f$. This notion is useful in other situations as well. At the end of the paper we collect a few open questions.

I would like to thank Marie-Françoise Roy and Ronan Quarez for stimulating discussions. In particular, the results of Section 3 were prompted by a question of Roy.

\section{DESCENDING SUMS OF SQUARES REPRESENTATIONS IN TOTALLY REAL EXTENSIONS}

Let $f \in \mathbb{Q}\left[x_{1}, \ldots, x_{n}\right]=\mathbb{Q}[\mathrm{x}]$ be a polynomial, and assume that $f$ is a sum of squares of polynomials in $K[\mathrm{x}]$ where $K$ is a real number field. In this section we review the result of Hillar [7] according to which $f$ is a sum of squares in $\mathbb{Q}[\mathrm{x}]$. We will show that it is a simple consequence of properties of the trace form of $K / \mathbb{Q}$. As a consequence, we will generalize the bound of Quarez [11] to the case where $K / \mathbb{Q}$ is not necessarily Galois.

1.1. Before giving the actual proof, which is very short, we need to recall a few facts about trace quadratic forms. Let $K / k$ be a finite separable field extension of degree $d:=[K: k]$, and consider the quadratic form

$$
\tau: K \rightarrow k, \quad y \mapsto \operatorname{tr}_{K / k}\left(y^{2}\right)
$$

over $k$, where $\operatorname{tr}_{K / k}$ denotes the trace of $K$ over $k$. The trace form $\tau$ has the following well-known property: For any ordering $P$ of $k$, the Sylvester signature of $\tau$ with respect to $P$ is equal to the number of extensions of the ordering $P$ to $K$. See [13, Lemma 3.2.7 or Theorem 3.4.5.

Assume that $k$ is real and that every ordering of $k$ has $d=[K: k]$ different extensions to $K$, or equivalently, that every ordering of $k$ extends to the Galois hull of $K$ over $k$. Then $\tau$ is positive definite with respect to every ordering of $k$. Diagonalizing $\tau$ therefore gives sums of squares $a_{1}, \ldots, a_{d}$ in $k^{*}$, together with a 
$k$-linear basis $y_{1}, \ldots, y_{d}$ of $K$, such that

$$
\operatorname{tr}_{K / k}\left(\left(\sum_{i=1}^{d} x_{i} y_{i}\right)^{2}\right)=\sum_{i=1}^{d} a_{i} x_{i}^{2}
$$

holds for all $x_{1}, \ldots, x_{d} \in k$. Note that we can choose $a_{1}=d$ here by starting the diagonalization with $y_{1}=1$. More generally, if $A$ is an arbitrary (commutative) $k$-algebra and $A_{K}=A \otimes_{k} K$, then

$$
\operatorname{tr}_{A_{K} / A}\left(\left(\sum_{i=1}^{d} x_{i} \otimes y_{i}\right)^{2}\right)=\sum_{i=1}^{d} a_{i} x_{i}^{2}
$$

holds for all $x_{1}, \ldots, x_{n} \in A$.

The following theorem is now a simple observation. It sharpens the results of Hillar [7] and Quarez [11:

Theorem 1.2. Let $K / k$ be an extension of real fields of degree $d=[K: k]<$ $\infty$, and assume that every ordering of $k$ extends to $d$ different orderings of $K$. Then there exist sums of squares $c_{1}, \ldots, c_{d}$ in $k$ with $c_{1}=1$ and with the following property:

For every $k$-algebra $A$, every $m \geq 1$ and every $f \in A$ which is a sum of $m$ squares in $A_{K}=A \otimes_{k} K$, there exist $f_{1}, \ldots, f_{d} \in A$ such that each $f_{i}$ is a sum of $m$ squares in $A$, and such that

$$
f=\sum_{i=1}^{d} c_{i} f_{i}
$$

In particular, $f$ is a sum of $d m \cdot p(k)$ squares in A. (This number can be improved, see Remarks 1.3 below.)

Here $p(k)$ denotes the Pythagoras number of $k$, i. e., the smallest number $p$ such that every sum of squares in $k$ is a sum of $p$ squares in $k$. (If no such number $p$ exists one puts $p(k)=\infty$.)

Proof. Choose sums of squares $a_{i}$ in $k$ and elements $y_{i} \in K(i=1, \ldots, d)$ as in 1.1 . It suffices to take $c_{i}=\frac{a_{i}}{d}$ for $i=1, \ldots, d$. Indeed, assuming $f=g_{1}^{2}+\cdots+g_{m}^{2}$ with $g_{1}, \ldots, g_{m} \in A_{K}$, we get

$$
d \cdot f=\operatorname{tr}_{A_{K} / A}(f)=\sum_{j=1}^{m} \operatorname{tr}_{A_{K} / A}\left(g_{j}^{2}\right)=\sum_{j=1}^{m} \sum_{i=1}^{d} a_{i} x_{i j}^{2},
$$

where the $x_{i j} \in A$ are determined by $g_{j}=\sum_{i=1}^{d} x_{i j} \otimes y_{i}(j=1, \ldots, m)$. So the assertion in the theorem holds with $f_{i}=\sum_{j=1}^{m} x_{i j}^{2}(i=1, \ldots, d)$.

Remarks 1.3. 1. The proof is completely constructive: Knowing the sums of squares decomposition of $f$ in $A_{K}$, we explicitly get $f_{1}, \ldots, f_{d}$ together with sums of squares decompositions in $A$.

2. Assume that $k$ is a number field, so $p(k)=4$. Using the well-known composition formulas for sums of four squares, we can improve the upper bound $4 d m$ in Theorem 1.2. Indeed, $c_{i} f_{i}$ is a sum of $4\left\lceil\frac{m}{4}\right\rceil$ squares for every $i$, and is a sum of $m$ squares for $i=1$, so altogether $f$ is a sum of

$$
m+4(d-1) \cdot\left\lceil\frac{m}{4}\right\rceil
$$


squares in $A$. This is precisely the bound found by Quarez [11 in the case where $k=\mathbb{Q}$ and $K / \mathbb{Q}$ is Galois. Note that this bound lies between $d m$ and $d(m+3)-3$.

3. Similar as in the previous remark, we can improve the bound in Theorem 1.2 for arbitrary $K / k$, using composition. In this way we obtain the general bound

$$
8 d \cdot\left\lceil\frac{p(k)}{8}\right\rceil \cdot\left\lceil\frac{m}{8}\right\rceil
$$

for the number of squares in $A$, which is roughly $\frac{1}{8}$ of the bound mentioned in 1.2 If $\min \{p(k), m\}$ is at most 4 (resp. 2), we get a better valid bound by replacing the number 8 in (1.3) by 4 (resp. 2). By making use of the fact that $c_{1}=1$, all these bounds can still be improved a little more, similar as in the previous remark.

1.4. The qualitative part of the above result extends immediately to the following more general situation. For any commutative ring $B$, let $\Sigma B^{2}$ denote the set of sums of squares in $B$. Let $K / k$ be a field extension and let $A$ be a (commutative) $k$ algebra. Fix elements $h_{1}, \ldots, h_{r} \in A$, amd consider the so-called (pseudo) quadratic module

$$
M:=\left\{\sum_{i=1}^{r} s_{i} h_{i}: s_{1}, \ldots, s_{r} \in \Sigma A^{2}\right\}
$$

generated in $A$ by the $h_{i}$. Similarly, let

$$
M_{K}:=\left\{\sum_{i=1}^{r} t_{i} h_{i}: t_{1}, \ldots, t_{r} \in \Sigma A_{K}^{2}\right\}
$$

be the (pseudo) quadratic module generated by $M$ in $A_{K}=A \otimes_{k} K$. Then we have:

Proposition 1.5. In the above situation, if $K / k$ is a finite extension of real fields such that every ordering of $k$ extends to $[K: k]$ different orderings of $K$, we have $A \cap M_{K}=M$.

Proof. Let $t_{1}, \ldots, t_{r} \in \Sigma A_{K}^{2}$ be such that $f:=\sum_{i=1}^{r} t_{i} h_{i}$ lies in $A$. Taking the trace of $f$ gives

$$
f=\frac{1}{d} \sum_{i=1}^{r} \operatorname{tr}_{A_{K} / A}\left(t_{i}\right) h_{i}
$$

For any $i=1, \ldots, r$, the $\operatorname{trace} \operatorname{tr}_{A_{K} / A}\left(t_{i}\right)$ lies in $\Sigma A^{2}$, see 1.1. It follows that $f \in M$.

\section{Construction of COUnterexamples}

We construct a family of forms with rational coefficients which are sums of squares over $\mathbb{R}$ but not over $\mathbb{Q}$ :

Theorem 2.1. Let $n \geq 2$, and let $d \geq 4$ be an even number. There exists a form $f \in \mathbb{Q}\left[x_{0}, \ldots, x_{n}\right]$ of degree $d$ with the following properties:

(1) $f$ is irreducible over $\mathbb{Q}$, and decomposes into a product of $d$ linear forms over $\mathbb{C}$;

(2) $f$ is a sum of two squares in $\mathbb{R}\left[x_{0}, \ldots, x_{n}\right]$;

(3) $f$ is not a sum of any number of squares in $\mathbb{Q}\left[x_{0}, \ldots, x_{n}\right]$. 
For example,

$$
f=x_{0}^{4}+x_{0} x_{1}^{3}+x_{1}^{4}-3 x_{0}^{2} x_{1} x_{2}-4 x_{0} x_{1}^{2} x_{2}+2 x_{0}^{2} x_{2}^{2}+x_{0} x_{2}^{3}+x_{1} x_{2}^{3}+x_{2}^{4}
$$

is such a form.

2.2. To prove the theorem we consider the following setup. Let $K$ be a totally imaginary number field of degree $d=2 m$, let $E$ be the Galois hull of $K / \mathbb{Q}$, and let $G=\operatorname{Gal}(E / \mathbb{Q})$ (resp. $H=\operatorname{Gal}(E / K)$ ) be the Galois group of $E$ over $\mathbb{Q}$ (resp. of $E$ over $K)$. The group $G$ acts transitively on the set $\operatorname{Hom}(K, E)$ of embeddings $K \rightarrow E$ by

$$
\left({ }^{\sigma} \varphi\right)(\alpha)=\sigma(\varphi(\alpha)) \quad(\alpha \in K)
$$

$(\sigma \in G, \varphi \in \operatorname{Hom}(K, E))$, thereby identifying the $G$-set $\operatorname{Hom}(K, E)$ with $G / H$. Note that $|G / H|=d$. We fix an embedding $E \hookrightarrow \mathbb{C}$ and denote by $\tau \in G$ the restriction of complex conjugation to $E$. Since $K$ is totally imaginary, $\tau$ acts on $G / H$ without fixpoint.

2.3. We extend the $G$-action on $E$ to an action on $E[\mathrm{x}]=E\left[x_{0}, \ldots, x_{n}\right]$ by letting $G$ act on the coefficients. Let $l \in K[\mathrm{x}]$ be a linear form, and let $L \subseteq \mathbb{P}^{n}$ be the hyperplane $l=0$. We assume that the $d$ Galois conjugates of $L$ are in general position, that is, the intersection of any $r \leq n+1$ of them has codimension $r$ (the empty set is assigned the codimension $n+1)$. For example, this condition is satisfied when $\alpha$ is a primitive element for $K / \mathbb{Q}$ and

$$
l=\sum_{i=0}^{n} \alpha^{i} x_{i}
$$

as one sees by a Vandermonde argument. We consider the form

$$
f:=\prod_{\sigma H \in G / H}{ }^{\sigma} l=N_{K / \mathbb{Q}}(l)
$$

of degree $d$. Clearly, $f$ has rational coefficients and is irreducible over $\mathbb{Q}$. Moreover, since $\tau$ acts on $G / H$ without fixpoint, we can choose $m=\frac{d}{2} \operatorname{cosets} \sigma_{1} H, \ldots, \sigma_{m} H$ in $G / H$ which represent the $\tau$-orbits. Writing $l_{j}:={ }^{\sigma_{j}} l(j=1, \ldots, m)$ we therefore have

$$
f=\prod_{i=1}^{m} l_{i} \bar{l}_{i}
$$

where bar denotes coefficientwise complex conjugation. This shows that $f$ is a product of $m$ quadratic forms over $\mathbb{R}$, each of which is a sum of two squares over $\mathbb{R}$. In particular, $f$ is a sum of two squares in $\mathbb{R}[\mathrm{x}]$.

2.4. Let us label the $d=2 m$ hyperplanes ${ }^{\sigma} l=0(\sigma H \in G / H)$ by $L_{1}, \ldots, L_{d}$. By our assumption of general position, the $\left(\begin{array}{l}d \\ 2\end{array}\right)$ pairwise intersections $M_{i j}=L_{i} \cap L_{j}$ $(1 \leq i<j \leq d)$ are all distinct, and are linear subspaces of $\mathbb{P}^{n}$ of codimension two. Exactly $m$ of the $M_{i j}$ are conjugation-invariant, and they correspond to the $\tau$-orbits in $G / H$. We say that $M_{i j}$ is real if it is conjugation-invariant.

We now assume that the action of $G$ on $G / H$ is 2 -transitive. Then $G$ acts transitively on the set $\left\{M_{i j}: 1 \leq i<j \leq d\right\}$. We claim that $f$ cannot be a sum of squares of forms with rational coefficients. To see this, suppose

$$
f=p_{1}^{2}+\cdots+p_{r}^{2}
$$


where $p_{1}, \ldots, p_{r}$ are forms of degree $m$ in $\mathbb{Q}[\mathrm{x}]$. Each $p_{\nu}$ vanishes identically on the $m$ real intersections $M_{i j}$. By Galois invariance and by the transitivity assumption, the $p_{\nu}$ have to vanish identically on all $\left(\begin{array}{l}d \\ 2\end{array}\right)$ intersections $M_{i j}$. But there is no nonzero form of degree $m$ with this property. In fact, we have $m \leq d-2$ since $d=2 m \geq 4$, and there is not even any nonzero such form of degree $d-2$. This follows from the next lemma, which we state in a stronger version with a view to a later application:

Lemma 2.5. Let $k$ be a field and $\mathrm{x}=\left(x_{0}, \ldots, x_{n}\right)$ with $n \geq 2$, and let $l_{1}, \ldots, l_{d} \in$ $k[\mathrm{x}]$ be linear forms such that the hyperplanes $L_{i}=\left\{l_{i}=0\right\}(i=1, \ldots, d)$ are in general position. Let I be the vanishing ideal of $\bigcup_{1 \leq i<j \leq d} L_{i} \cap L_{j}$ in $k[\mathrm{x}]$. Then $I$ is generated by the $d$ forms

$$
p_{i}:=\frac{l_{1} \cdots l_{d}}{l_{i}} \quad(i=1, \ldots, d)
$$

of degree $d-1$.

In particular, for $d \geq 3$ there is no hypersurface of degree $d-2$ containing $L_{i} \cap L_{j}$ for all $1 \leq i<j \leq d$.

Proof. The assertion is obviously true for $d \leq 2$, so we can assume that $d \geq 3$ and the lemma is proved for smaller values of $d$. Clearly we have $\left(p_{1}, \ldots, p_{d}\right) \subseteq I$. Conversely let $g \in I$ be a form. Since $L_{1} \cap L_{2}, \ldots, L_{1} \cap L_{d}$ are distinct hypersurfaces in $L_{1}$, and since $g$ vanishes on all of them, we see that $g$ is a multiple of $l_{2} \cdots l_{d}=p_{1}$ modulo $l_{1}$, that is, $g=g_{1} p_{1}+l_{1} h$ with suitable forms $g_{1}$ and $h$. The form $h$ vanishes on the pairwise intersections of the hypersurfaces $L_{2}, \ldots, L_{d}$. Writing $q_{i}:=\left(l_{2} \cdots l_{d}\right) / l_{i}$ for $i=2, \ldots, d$, it follows from the inductive hypothesis that $h \in\left(q_{2}, \ldots, q_{d}\right)$. Since $l_{1} q_{i}=p_{i}$ for $i=2, \ldots, d$, we conclude $g \in\left(p_{1}, \ldots, p_{d}\right)$, as desired.

Let $\overline{\mathbb{Q}}$ denote an algebraic closure of $\mathbb{Q}$. Summarizing, we have proved:

Theorem 2.6. Let $n \geq 2$, let $K / \mathbb{Q}$ be a totally imaginary number field of degree $d \geq 4$, and let $l \in K\left[x_{0}, \ldots, x_{n}\right]$ be a linear form whose $d$ Galois conjugates over $\mathbb{Q}$ are in general position. If the action of $\operatorname{Gal}(\overline{\mathbb{Q}} / \mathbb{Q})$ on $\operatorname{Hom}(K, \mathbb{C})$ is 2-transitive, then

$$
f:=N_{K / \mathbb{Q}}(l)
$$

is a form of degree $d$ with rational coefficients that is irreducible over $\mathbb{Q}$ and a sum of two squares over $\mathbb{R}$, but not a sum of any number of squares over $\mathbb{Q}$.

2.7. Clearly, this implies the statement of Theorem 2.1. We may start with any totally imaginary number field $K$ of degree $d \geq 4$ for which the Galois action on $\operatorname{Hom}(K, \mathbb{C})$ is 2 -transitive. For example, the Galois group may act as the alternating or full symmetric group on $d$ letters. Picking any primitive element $\alpha$ of $K / \mathbb{Q}$, the form $f$ constructed as in 2.3 satisfies all the properties of 2.1

Example 2.8. To produce an explicit example, take the field $K=\mathbb{Q}(\alpha)$ where $\alpha^{4}-\alpha+1=0$. In this case the Galois group acts as the full symmetric group on the roots of $t^{4}-t+1$, as one sees by reducing modulo 2 and modulo 3. Starting with $l=x_{0}+\alpha x_{1}+\alpha^{2} x_{2}$, one obtains the form $f$ displayed after Theorem 2.1. 
To see a sum of squares representation of $f$ explicitly, let $\beta$ be a root of $t^{3}-4 t-1=$ 0 (the cubic resolvent of $t^{4}-t+1$ ). Then the following decomposition holds:

$4 f=\left(2 x_{0}^{2}+\beta x_{1}^{2}-x_{1} x_{2}+\left(2+\frac{1}{\beta}\right) x_{2}^{2}\right)^{2}-\beta\left(2 x_{0} x_{1}-\frac{x_{1}^{2}}{\beta}+\frac{2 x_{0} x_{2}}{\beta}+\beta x_{1} x_{2}-x_{2}^{2}\right)^{2}$.

The cubic field $\mathbb{Q}(\beta)$ is totally real, but not Galois over $\mathbb{Q}$. Its three places send $\beta$ to real numbers approximately equal to

$$
-1.860805854, \quad-0.2541016885, \quad 2.114907542,
$$

respectively. Therefore, the first two embeddings give each a representation of $f$ as a sum of two squares of real quadratic forms. These representations are defined over the real field $F=\mathbb{Q}(\sqrt{-\beta})$ of degree six. Up to equivalence, these are the only two representations of $f$ over $\mathbb{R}$ as a sum of two squares. Every other sum of squares representation of $f$ over $\mathbb{R}$ is (equivalent to) a sum of four squares, and arises as a convex combination of the two extremal representations.

Remark 2.9. For the conclusion of Theorem 2.6, it is not necessary that $G$ acts 2transitively on $G / H$, or equivalently, that $G$ acts transitively on the set $\left\{M_{i j}: 1 \leq\right.$ $i<j \leq d\}$ (see 2.4). It suffices that any $G$-orbit in this set contains at least one space $M_{i j}$ which is real, i.e., invariant under complex conjugation $\tau$. In terms of the $G$-action on $G / H$, this means the following condition:

(*) For any $x, y \in G / H$ with $x \neq y$ there exist $z \in G / H$ and $\sigma \in G$ such that $x=\sigma z$ and $y=\sigma \tau z$.

For $d=|G / H|=4$, condition (*) implies 2-transitivity of $G$ on $G / H$. But for $d \geq 6$ there are examples where $G$ satisfies $(*)$ without being 2 -transitive. The simplest such example is given by the group $G$ of rotations of a regular cube $P$, acting on the set $F$ of (two-dimensional) faces. So $G=S_{4}$, the symmetric group on four letters, and $H$ is the cyclic subgroup generated by a 4 -cycle in $G$. A pair $\left\{f, f^{\prime}\right\}$ of different faces of $P$ consists either of two faces with a common edge, or of two opposite faces. Hence there are exactly two $G$-orbits in the $\operatorname{set}\left(\begin{array}{c}F \\ 2\end{array}\right)$ of pairs of faces. The involution $\tau$ is the rotation of order two around an axis that joins the midpoints of two opposite edges. Among the three pairs $\{f, \tau f\}(f \in F)$ of faces, one consists of opposite faces, while the other two consist of adjacent faces. So each pair of faces is $G$-conjugate to a pair of the form $\{f, \tau f\}$.

An example of a (totally imaginary) number field which realizes this Galois action on its set of places is $k=\mathbb{Q}(\alpha)$ with

$$
\alpha^{6}-\alpha^{5}+2 \alpha^{4}+\alpha^{3}+2 \alpha^{2}+3 \alpha+1=0 .
$$

The example was found by consulting the Bordeaux number field tables [4.

Remark 2.10. We can easily extend Theorem 2.1 to real number fields other than $\mathbb{Q}$. Indeed, let $K, E, l, f$ etc. be as in 2.3 , and assume that $G=\operatorname{Gal}(E / \mathbb{Q})$ acts 2 -transitively on $\operatorname{Hom}(K, E)$. Let $k$ be any number field with at least one real place, and consider the natural embedding $\phi$ from $\operatorname{Gal}(k E / k)$ into $G$, induced by restriction of automorphisms. Then $\phi$ is surjective if (and only if) $E \cap k=\mathbb{Q}$, that is, if $E$ and $k$ are linearly disjoint over $\mathbb{Q}$. Assuming that this is the case, we claim that $f$ is not a sum of squares over $k$. Indeed, by the argument in 2.4, the $\left(\begin{array}{l}d \\ 2\end{array}\right)$ intersections $M_{i j}=L_{i} \cap L_{j}$ are all Galois conjugate among each other over $k$. If there were an identity $f=p_{1}^{2}+\cdots+p_{r}^{2}$ with forms $p_{\nu} \in k[\mathrm{x}]$, the $p_{\nu}$ would have to vanish on the union of the $M_{i j}$, which again is impossible by Proposition 2.5. 
Using this way of reasoning, we conclude:

Corollary 2.11. Let $k$ be any fixed number field with at least one real place, let $n \geq 2$ and $d \geq 4$ be even. Then there exists a form $f \in \mathbb{Q}\left[x_{0}, \ldots, x_{n}\right]$ of degree $d$ which is a sum of two squares of forms over $\mathbb{R}$, but not a sum of squares of forms over $k$.

In particular, over a real number field there is no analogue of Hilbert's theorem 6] over $\mathbb{R}$, according to which every nonnegative ternary quartic form is a sum of squares of quadratic forms.

Proof. Given $k$, it suffices by 2.10 to find a totally imaginary extension $K / \mathbb{Q}$ with Galois hull $E / \mathbb{Q}$ for which $G=\operatorname{Gal}(E / \mathbb{Q})$ acts 2-transitively on $\operatorname{Hom}(K, E)$, and such that $E$ and $k$ are linearly disjoint. The latter will certainly be the case if the discriminants of $E$ and $k$ are relatively prime. So the assertion follows from the next lemma.

Lemma 2.12. For any finite set $S$ of primes and any even number $d$, there exists a totally imaginary number field $K / \mathbb{Q}$ of degree $d$ with Galois hull $E / \mathbb{Q}$, such that the discriminant of $E$ is not divisible by any prime in $S$, and such that $\operatorname{Gal}(E / \mathbb{Q})$ is 2-transitive on $\operatorname{Hom}(K, E)$.

Proof. It suffices to find a monic polynomial $g(x)$ over $\mathbb{Z}$ of degree $d$ with the following properties: (1) $g$ is positive definite; (2) the discriminant of $g$ is not divisible by any prime in $S$; (3) there exist primes $p, q$ such that $g \bmod p$ is irreducible and $g \bmod q$ is a linear factor times an irreducible polynomial. Given such $g$, let $K=\mathbb{Q}(\alpha)$ where $\alpha$ is a root of $g$, and let $E$ be the Galois hull of $K$. Then $K$ has the required properties. In particular, the action of $G=\operatorname{Gal}(E / \mathbb{Q})$ on the roots of $g$ is 2-transitive since $G$ contains a $(d-1)$-cycle. Properties $(2)$ and $(3)$ can be guaranteed by arranging a particular factor decomposition of $g$ modulo $p$, for finitely many primes $p$. So it is clear that (many) polynomials $g$ as above can be found.

\section{Rational DENOMinators}

3.1. Let $\mathrm{x}=\left(x_{0}, \ldots, x_{n}\right)$ with $n \geq 2$, and let $f \in \mathbb{Q}[\mathrm{x}]$ be a form of degree $d$ as constructed in Theorem 2.1. In particular, $f$ is a sum of squares over $\mathbb{R}$, but not over $\mathbb{Q}$. By Artin's solution 3] to Hilbert's 17th problem, $f$ is a sum of squares of rational functions over $\mathbb{Q}$. In other words, there exists a form $h \neq 0$ in $\mathbb{Q}[\mathrm{x}]$ such that both $h$ and $f h$ are sums of squares over $\mathbb{Q}$. When $f$ is constructed explicitly as in Section 2, what can be said about the degree of such $h$ ? Is it possible to give explicit constructions for $h$ ?

These questions were raised by M.-F. Roy. We will give a complete answer for $d=4$, and a partial answer for $d \geq 6$, see Theorem 3.3 and Proposition 3.4

3.2. For the following we assume the setup of Theorem 2.6 . Hence $K / \mathbb{Q}$ will be a totally imaginary number field of degree $d \geq 4$, with Galois hull $E / \mathbb{Q}$. Letting $G=\operatorname{Gal}(E / \mathbb{Q})$ and $H=\operatorname{Gal}(E / H)$, we assume that the action of $G$ on $G / H$ is 2 -transitive. (In fact, it will suffice for the following to have the weaker condition 2.9 satisfied.) Let $l \in K[\mathrm{x}]$ be a linear form such that the $d$ Galois conjugates of the hyperplane $l=0$ are in general position. The form

$$
f=\prod_{\sigma H \in G / H}{ }^{\sigma} l=N_{K / \mathbb{Q}}(l)
$$


in $\mathbb{Q}[\mathrm{x}]$ satisfies the conclusions of Theorem 2.6.

Theorem 3.3. Let $f$ be as in 3.2.

(a) There exists a nonzero form $h \in \mathbb{Q}[\mathrm{x}]$ of degree $d-2$, but not of smaller degree, for which $f h$ is a sum of squares of forms in $\mathbb{Q}[\mathrm{x}]$.

(b) When $d=4$, any $h$ as in (a) is a sum of squares of linear forms in $\mathbb{Q}[\mathrm{x}]$.

Proof. Let $0 \neq h \in \mathbb{Q}[\mathrm{x}]$ be a form for which $f h$ is a sum of squares of forms over $\mathbb{Q}$, say $f h=g_{1}^{2}+\cdots+g_{r}^{2}$ with forms $g_{1}, \ldots, g_{r} \in \mathbb{Q}[\mathrm{x}]$. Let $l=l_{1}, l_{2}, \ldots, l_{d} \in \overline{\mathbb{Q}}[\mathrm{x}]$ be the linear forms that are Galois conjugate to $l$, let $L_{i}$ be the hyperplane $l_{i}=0$, and consider the pairwise intersections $L_{i} \cap L_{j}(1 \leq i<j \leq d)$. The forms $g_{\nu}$ vanish identically on those intersections $L_{i} \cap L_{j}$ that are real (there are $\frac{d}{2}$ such). Since the action of $G$ permutes the $L_{i} \cap L_{j}$ transitively, and since the $g_{\nu}$ have rational coefficients, the $g_{\nu}$ vanish identically on $\bigcup_{i<j} L_{i} \cap L_{j}$. The vanishing ideal $I$ of this union (inside $\overline{\mathbb{Q}}[\mathrm{x}]$ ) is generated by the forms $p_{i}:=\frac{f}{l_{i}}(i=1, \ldots, d)$, by Lemma 2.5 We already conclude that $\operatorname{deg}\left(g_{\nu}\right) \geq d-1$, and hence $\operatorname{deg}(h) \geq d-2$.

It remains to construct a form $h$ of exact degree $d-2$ for which $f h$ is a sum of squares over $\mathbb{Q}$. Note that assertion (b) is clear from (a), since here $h$ is a quadratic form over $\mathbb{Q}$ and is nonnegative on $\mathbb{R}^{n+1}$.

The proof of Theorem 3.3 will therefore be completed by the next proposition. It gives a fully explicit rendering of the theorem:

Proposition 3.4. Let $f$ be as in 3.2. For a form $g \in \mathbb{Q}[\mathrm{x}]$ of degree $2 d-2$, the following conditions are equivalent:

(i) $g$ is divisible by $f$ and is a sum of squares of forms in $\mathbb{Q}[\mathrm{x}]$;

(ii) there exist $r \geq 1$ and elements $a_{1}, \ldots, a_{r} \in K$ with $a_{1}^{2}+\cdots+a_{r}^{2}=0$ such that

$$
g=\sum_{\nu=1}^{r}\left(\operatorname{tr}_{K / \mathbb{Q}}\left(\frac{a_{\nu} f}{l}\right)\right)^{2} .
$$

If (i) and (ii) hold, then conversely every sum of squares representation of $g$ in $\mathbb{Q}[\mathrm{x}]$ has the form stated in (ii), for suitable $a_{\nu} \in K$ with $\sum_{\nu} a_{\nu}^{2}=0$.

Proof. Assume $g=g_{1}^{2}+\cdots+g_{r}^{2}$ where $g_{1}, \ldots, g_{r} \in \mathbb{Q}[\mathrm{x}]$ are forms of degree $d-1$, and assume that $g$ is divisible by $f$. As before, let $I \subseteq \overline{\mathbb{Q}}[\mathrm{x}]$ be the vanishing ideal of $\bigcup_{i<j} L_{i} \cap L_{j}$. By Lemma 2.5 we have $g_{1}, \ldots, g_{r} \in I \cap \mathbb{Q}[\mathrm{x}]$.

Let $\operatorname{tr}=\operatorname{tr}_{K / \mathbb{Q}}$ be the trace of $K$ over $\mathbb{Q}$. We claim that a form $g \in I$ of degree $d-1$ has $\mathbb{Q}$-coefficients if and only if $g=\operatorname{tr}(a f / l)$ for some $a \in K$. Indeed, let

$$
g=b_{1} \frac{f}{l_{1}}+\cdots+b_{d} \frac{f}{l_{d}}
$$

with $b_{i} \in \overline{\mathbb{Q}}$. Let us label the elements of $G / H$ as $\sigma_{1} H, \ldots, \sigma_{d} H$ in such a way that $\sigma_{1}=1$ and $l_{i}={ }^{\sigma_{i}} l$ for $i=1, \ldots, d$. The forms $\frac{f}{l_{1}}, \ldots, \frac{f}{l_{d}}$ are linearly independent. We conclude that $g$ lies in $\mathbb{Q}[\mathrm{x}]$ if and only if $b_{1} \in K$ and $b_{i}=\sigma_{i}\left(b_{1}\right)$ for $i=1, \ldots, d$, or in other words, if and only if $g=\operatorname{tr}\left(b_{1} f / l\right)$ with $b_{1} \in K$.

It remains to characterize when a sum of squares

$$
g=\sum_{\nu=1}^{r}\left(\operatorname{tr}\left(\frac{a_{\nu} f}{l}\right)\right)^{2}
$$


with $a_{1}, \ldots, a_{r} \in K$ is divisible by $f=l_{1} \cdots l_{d}$, or equivalently, by $l=l_{1}$. Since

$$
g=\sum_{\nu=1}^{r}\left(\sigma_{1}\left(a_{\nu}\right) \frac{f}{l_{1}}+\cdots+\sigma_{d}\left(a_{\nu}\right) \frac{f}{l_{d}}\right)^{2}
$$

we see that $g$ is divisible by $l_{1}$ if and only if $\sum_{\nu} a_{\nu}^{2}=0$.

The proof of Proposition 3.4 and therefore of Theorem 3.3, is complete.

Remark 3.5. In (a) of Theorem [3.3. we can always find $0 \neq h \in \mathbb{Q}[\mathrm{x}]$ of degree $d-2$ such that $f h$ is a sum of five squares in $\mathbb{Q}[\mathrm{x}]$. This follows from 3.4 since -1 is a sum of four squares in $K$. If $K$ happens to have level 2, i.e., if -1 is a sum of two squares in $K$, then $f h$ can be made a sum of three squares in $\mathbb{Q}[\mathrm{x}]$. (Note that -1 cannot be a square in $K$, and so $f h$ cannot be made a sum of two squares in $\mathbb{Q}[\mathrm{x}]$.)

Example 3.6. To illustrate the preceding construction, let us review the example of a ternary quartic $f \in \mathbb{Q}\left[x_{0}, x_{1}, x_{2}\right]$ given after Theorem 2.1 (c.f. also Example 2.8). In this case, the number field $K=\mathbb{Q}(\alpha)$ with $\alpha^{4}-\alpha+1=0$ has level 2 , as one can conclude from general reasons since the prime 2 is inert in $K$. Explicitly, this is confirmed by the identity

$$
\left(\alpha^{2}+\alpha-1\right)^{2}+\left(\alpha^{2}-\alpha\right)^{2}+1=0
$$

in $K$. Writing

$$
g_{1}=\operatorname{tr}\left(\frac{f}{l}\right), \quad g_{2}=\operatorname{tr}\left(\frac{\left(\alpha^{2}+\alpha-1\right) f}{l}\right), \quad g_{3}=\operatorname{tr}\left(\frac{\left(\alpha^{2}-\alpha\right) f}{l}\right)
$$

with $\beta=\alpha^{2}+\alpha-1$ and $\gamma=\alpha^{2}-\alpha$, we find

$$
\begin{aligned}
& g_{1}=4 x_{0}^{3}+x_{1}^{3}+x_{2}^{3}+4 x_{0} x_{2}^{2}-4 x_{1}^{2} x_{2}-6 x_{0} x_{1} x_{2}, \\
& g_{2}=-4 x_{0}^{3}+3 x_{1}^{3}+4 x_{2}^{3}-3 x_{0}^{2} x_{1}-x_{0} x_{1}^{2}+x_{0}^{2} x_{2}-x_{0} x_{2}^{2}+4 x_{1}^{2} x_{2}+3 x_{1} x_{2}^{2}-2 x_{0} x_{1} x_{2}, \\
& g_{3}=-4 x_{1}^{3}+3 x_{2}^{3}-3 x_{0}^{2} x_{1}-7 x_{0} x_{1}^{2}+7 x_{0}^{2} x_{2}+3 x_{0} x_{2}^{2}+3 x_{1} x_{2}^{2}+8 x_{0} x_{1} x_{2} .
\end{aligned}
$$

Expanding the sum of squares, we obtain

$$
f h=g_{1}^{2}+g_{2}^{2}+g_{3}^{2}
$$

where

$$
h=32 x_{0}^{2}+24 x_{0} x_{1}-8 x_{0} x_{2}+26 x_{1}^{2}+16 x_{1} x_{2}+26 x_{2}^{2} .
$$

To write $h$ as a sum of squares in an explicit way, we may observe

$$
86 h=43\left(8 x_{0}+3 x_{1}-x_{2}\right)^{2}+\left(43 x_{1}+19 x_{2}\right)^{2}+1832 x_{2}^{2} .
$$

\section{TERnARY QUARTICS}

In this section we restrict to ternary forms of degree four. It was proved by Hilbert in 1888 [ 6] that every nonnegative quartic form $f \in \mathbb{R}\left[x_{0}, x_{1}, x_{2}\right]$ is a sum of squares of quadratic forms (and, in fact, of three squares). In Theorem 2.1 we constructed a family of quartic forms $f \in \mathbb{Q}\left[x_{0}, x_{1}, x_{2}\right]$ that are sums of squares over $\mathbb{R}$, but not over $\mathbb{Q}$. Now we'll show that conversely every nonnegative ternary quartic $f \in \mathbb{Q}\left[x_{0}, x_{1}, x_{2}\right]$ that fails to be a sum of squares over $\mathbb{Q}$ arises from the construction in 2.1. More precisely, we'll prove: 
Theorem 4.1. Let $f \in \mathbb{Q}\left[x_{0}, x_{1}, x_{2}\right]$ be a nonnegative form of degree 4 which is not a sum of squares over $\mathbb{Q}$. Then $f$ is a product $f=l_{1} l_{2} l_{3} l_{4}$ of linear forms in $\mathbb{C}\left[x_{0}, x_{1}, x_{2}\right]$, the four lines $l_{i}=0$ are in general position, and $\mathrm{Gal}(\overline{\mathbb{Q}} / \mathbb{Q})$ acts on the set of these lines as the symmetric or alternating group on four letters.

Before starting the proof, we need to introduce an important general concept. In the sequel let $\mathrm{x}=\left(x_{0}, \ldots, x_{n}\right)$ with arbitrary $n \geq 1$, and denote by $\Sigma$ the cone of sums of squares in $\mathbb{R}[\mathrm{x}]$.

Definition 4.2. Given a sum of squares $f \in \Sigma$, the set

$$
U_{f}:=\left\{p \in \mathbb{R}[\mathrm{x}]: f-\varepsilon p^{2} \in \Sigma \text { for some } \varepsilon>0\right\}
$$

will be called the characteristic subspace for $f$.

Lemma 4.3. Let $f \in \Sigma$.

(a) The set $\left\{p \in \mathbb{R}[\mathrm{x}]: f-p^{2} \in \Sigma\right\}$ is convex. Hence $U_{f}$ is a linear subspace of $\mathbb{R}[\mathrm{x}]$.

(b) There is a sum of squares representation $f=p_{1}^{2}+\cdots+p_{r}^{2}$ of $f$ in which $p_{1}, \ldots, p_{r}$ is a linear basis of $U_{f}$.

Proof. If $f-p_{j}^{2} \in \Sigma$ for $j=1,2$, then

$$
f-\left((1-t) p_{1}+t p_{2}\right)^{2}=(1-t)\left(f-p_{1}^{2}\right)+t\left(f-p_{2}^{2}\right)+t(1-t)\left(p_{1}-p_{2}\right)^{2} \in \Sigma
$$

for $0 \leq t \leq 1$, proving (a). As for (b), there is a basis $q_{1}, \ldots, q_{r}$ of $U_{f}$ such that $f-q_{i}^{2} \in \Sigma$ for $i=1, \ldots, r$. By averaging over corresponding sums of squares expressions we find a sum of squares representation $f=g_{1}^{2}+\cdots+g_{k}^{2}$ in which $g_{1}, \ldots, g_{k} \operatorname{span} U_{f}$. Now diagonalizing the symmetric tensor $\sum_{i=1}^{k} g_{i} \otimes g_{i} \in U_{f} \otimes U_{f}$ gives the assertion.

The reason why the characteristic subspaces will be useful here is the following lemma (which generalizes [7] Theorem 1.2):

Lemma 4.4. Let $f \in \mathbb{Q}[\mathrm{x}] \cap \Sigma$, i.e., $f$ is a polynomial with rational coefficients and is a sum of squares in $\mathbb{R}[\mathrm{x}]$. If the subspace $U_{f}$ of $\mathbb{R}[\mathrm{x}]$ is defined over $\mathbb{Q}$, then $f$ is a sum of squares in $\mathbb{Q}[\mathrm{x}]$.

(If $V$ is a $\mathbb{Q}$-vector space, a linear subspace $L$ of $V \otimes \mathbb{Q} \mathbb{R}$ is said to be defined over $\mathbb{Q}$ if it is spanned by $L \cap V$. Similarly for affine-linear subspaces.)

Proof. Let $U_{f} \subseteq \mathbb{R}[\mathrm{x}]$ be the characteristic subspace of $f$, let $S^{2} U_{f}$ be its second symmetric power, and let $\gamma: S^{2} U_{f} \rightarrow \mathbb{R}[\mathrm{x}]$ be the natural linear (product) map. Since $U_{f}$ is defined over $\mathbb{Q}$, so is $\Gamma_{f}:=\gamma^{-1}(f)$, an affine-linear subspace of $S^{2} U_{f}$. By Lemma 4.3(b), $\Gamma_{f}$ contains an element of $S^{2} U_{f}$ that is positive definite. From density of $\mathbb{Q}$ in $\mathbb{R}$ we conclude that $\Gamma_{f}$ also contains a positive definite element defined over $\mathbb{Q}$. In particular, hence, $f$ is a sum of squares over $\mathbb{Q}$.

Hilbert's theorem [6] on ternary quartics allows us to give an easy geometric descriptions for the characteristic subspaces of ternary quartics. First, the problem is local:

Lemma 4.5. Let $f, g \in \mathbb{R}[\mathrm{x}]$ be two nonnegative forms of the same degree. Assume for every $0 \neq \xi \in \mathbb{R}^{n+1}$ that there exists $\varepsilon>0$ for which $f-\varepsilon g$ is nonnegative in a neighborhood of $\xi$. Then there exists $\varepsilon>0$ such that $f-\varepsilon g$ is nonnegative on $\mathbb{R}^{n+1}$. 
Proof. This follows from compactness of projective space: For any $\xi \in \mathbb{P}^{n}(\mathbb{R})$ there exists $\varepsilon_{\xi}>0$ and a neighborhood $W_{\xi} \subseteq \mathbb{P}^{n}(\mathbb{R})$ of $\xi$ such that $f-\varepsilon_{\xi} g$ is nonnegative on $W_{\xi}$. Choose finitely many points $\xi_{1}, \ldots, \xi_{r} \in \mathbb{P}^{n}(\mathbb{R})$ such that $\mathbb{P}^{n}(\mathbb{R})=\bigcup_{i=1}^{r} W_{\xi_{i}}$, and put $\varepsilon=\min \left\{\varepsilon_{\xi_{i}}: i=1, \ldots, r\right\}$. Then $f-\varepsilon g$ is everywhere nonnegative.

From now on let $\mathrm{x}=\left(x_{0}, x_{1}, x_{2}\right)$. For a nonnegative ternary quartic $f \in \mathbb{R}[\mathrm{x}]$ with isolated real zeros, we will determine the characteristic subspace $U_{f}$ explicitly. (The case where the real zeros are not isolated is even easier, since it reduces to nonnegative quadratic forms.) By Lemma 4.5, it suffices to do this locally, namely to determine the subspace

$$
U_{f, \xi}:=\left\{p \in \mathbb{R}[\mathrm{x}]_{2}: \exists \varepsilon>0 f-\varepsilon p^{2} \geq 0 \text { around } \xi\right\}
$$

for every $\xi \in \mathbb{P}^{2}(\mathbb{R})$ with $f(\xi)=0$. Note that $U_{f, \xi}$ is also the space of all $p \in \mathbb{R}[\mathrm{x}]_{2}$ for which $p^{2} / f$ is locally bounded around $\xi$ in $\mathbb{P}^{2}(\mathbb{R})$. (Here $\mathbb{R}[\mathrm{x}]_{2}$ denotes the space of quadratic forms in $\mathbb{R}[\mathrm{x}]$.)

Assume that $\xi$ is an isolated real zero of a quartic form $f \in \mathbb{R}[\mathrm{x}]$. Then $\xi$ is a singularity of the curve $f=0$ of real type $A_{1}^{*}, A_{3}^{*}, A_{5}^{*}, A_{7}^{*}$ or $X_{9}^{* *}$. The last two can occur only when $f$ is reducible over $\mathbb{C}$. Here, by a (plane) $A_{k}^{*}$-singularity (for $k \geq 1$ odd), we mean a real analytic singularity of type $A_{k}$ whose two analytic branches are complex conjugate. See 4.7 for $X_{9}^{* *}$.

Proposition 4.6. Let $f(x, y), p(x, y)$ be real analytic function germs in $\left(\mathbb{R}^{2}, 0\right)$, and assume that the singularity $f=0$ is of type $A_{2 r-1}^{*}$ with $r \geq 1$. Then the germ $p^{2} / f$ is locally bounded in $\mathbb{R}^{2}$ around 0 if and only if $i(f, p) \geq 2 r$, where $i$ denotes the local intersection number at $0 \in \mathbb{R}^{2}$.

Proof. We may assume $f=y^{2}+x^{2 r}$, and we'll show that both properties are equivalent to $\omega(p(x, 0)) \geq r$, where $\omega$ is the vanishing order at $x=0$. It is clear that $\frac{p^{2}}{f}$ locally bounded implies $\omega(p(x, 0)) \geq r$. Conversely, if $\omega(p(x, 0)) \geq r$, we can write $p=y g+x^{r} h$ with analytic germs $g, h$. Then a simple calculation shows that $\frac{p^{2}}{f}$ is locally bounded around $0 \in \mathbb{R}^{2}$. On the other hand, from $f=\left(y+i x^{r}\right)\left(y-i x^{r}\right)$ we can directly deduce that $\omega(p(x, 0)) \geq r$ if and only if $i(f, p) \geq 2 r$.

Remark 4.7. A plane real singularity of type $X_{9}^{* *}$ corresponds to the union of four nonreal lines through a real point (two pairs of complex conjugate lines), see 2 . p. 185. A normal form is given by $f=x^{4}+y^{4}+a x^{2} y^{2}$ with $a>0, a \neq 2$. For a real analytic germ $p(x, y)$, the quotient $\frac{p^{2}}{f}$ is locally bounded iff $\omega(p(x, y)) \geq 2$ iff $i(f, p) \geq 8$.

Corollary 4.8. Let $f \in \mathbb{Q}[\mathrm{x}]$ be a nonnegative ternary quartic over $\mathbb{Q}$, let $\xi_{1}, \ldots, \xi_{r}$ be isolated real zeros of $f$ in $\mathbb{P}^{2}$, and assume that the set $\left\{\xi_{1}, \ldots, \xi_{r}\right\}$ is invariant under the action of $\operatorname{Gal}(\overline{\mathbb{Q}} / \mathbb{Q})$ on $\mathbb{P}^{2}(\overline{\mathbb{Q}})$. Then the subspace $\bigcap_{j=1}^{r} U_{f, \xi_{j}}$ of $\mathbb{R}[\mathrm{x}]_{2}$ is defined over $\mathbb{Q}$.

Note that $\xi_{1}, \ldots, \xi_{r}$ have coordinates in $\overline{\mathbb{Q}}$, so the Galois group acts on these points.

Proof. By Hilbert's theorem [6], this is clear from 4.6 and 4.7.

4.9. We now give the proof of Theorem 4.1. Let $\mathrm{x}=\left(x_{0}, x_{1}, x_{2}\right)$, let $f \in \mathbb{Q}[\mathrm{x}]$ be a nonnegative form of degree 4 . Let $U_{f} \subseteq \mathbb{R}[\mathrm{x}]_{2}$ be the characteristic subspace of 
$f$ (see 4.2). By a case distinction we will show that $f$ is a sum of squares over $\mathbb{Q}$ unless it satisfies the conditions of Theorem 4.1 .

Whenever $U_{f}$ is defined over $\mathbb{Q}, f$ is a sum of squares over $\mathbb{Q}$ by Lemma 4.4. In particular, this is the case when $f$ is strictly positive definite, since then $U_{f}=\mathbb{R}[\mathrm{x}]_{2}$. So we assume that $f$ has at least one real zero. We first consider the case where $f$ is absolutely irreducible. The real zeros of $f$ are precisely the real singular points of the curve $f=0$. The configuration of all (real or nonreal) singularities of this curve is one of the following (see [14] 7.3):

$$
A_{1}^{*}, 2 A_{1}^{*}, 3 A_{1}^{*}, A_{3}^{*}, A_{1}^{*}+A_{3}^{*}, A_{5}^{*}, A_{1}^{*}+2 A_{1}^{i}, A_{1}^{*}+2 A_{2}^{i} .
$$

(Here $2 A_{k}^{i}$ denotes a pair $P \neq \bar{P}$ of complex conjugate $A_{k}$-singularities.) The singularities are permuted by the Galois action. In all cases except the last two, every singularity of $f$ is real. By Lemma 4.5 and Corollary 4.8, the subspace $U_{f}$ is defined over $\mathbb{Q}$ in these cases, and we are done. In the case of $A_{1}^{*}+2 A_{2}^{i}$, the same is true since the unique real singularity is Galois invariant, hence defined over $\mathbb{Q}$.

It remains to consider the case where $f$ has three nodes, one of which is real (with a pair of nonreal tangents) and the other two are complex conjugate. Here $U_{f}$ consists of the quadratic forms with a zero in the real node, and we see that $U_{f}$ fails to be defined over $\mathbb{Q}$. Instead we can argue as follows: For such $f$, there exists a unique (up to orthogonal equivalence) representation $f=p_{1}^{2}+p_{2}^{2}+p_{3}^{2}$ in $\mathbb{C}[\mathrm{x}]$ for which $p_{1}, p_{2}, p_{3}$ vanish in all three nodes. Moreover, the symmetric tensor $t:=\sum_{j=1}^{3} p_{j} \otimes p_{j}$ is defined over $\mathbb{R}$ and is positive semidefinite. This follows from the analysis in 14 (for more details see [15, pp. 4 and 6). Since the set of all three nodes is Galois invariant, the tensor $t$ is defined over $\mathbb{Q}$, and hence $f$ is a sum of squares over $\mathbb{Q}$. We have thus shown that $f$ is a sum of squares over $\mathbb{Q}$ when $f$ is absolutely irreducible.

4.10. It is easily seen that $f$ is a sum of squares over $\mathbb{Q}$ whenever $f$ is reducible over $\mathbb{Q}$. Hence we can assume that $f$ is irreducible over $\mathbb{Q}$, but reducible over $\mathbb{C}$. So $f$ is either the $K / \mathbb{Q}$-norm of a quadratic form $p \in K[\mathrm{x}]$ defined over a quadratic field $K / \mathbb{Q}$, or the $K / \mathbb{Q}$-norm of a linear form $l \in K[\mathrm{x}]$ defined over a field $K$ of degree 4. In either case, $K$ is generated by the coefficients of $f$. First consider the case $[K: \mathbb{Q}]=2$. It is clear that $f$ is a sum of squares over $\mathbb{Q}$ when $K$ is imaginary. When $K$ is real, both $p$ and its $K / \mathbb{Q}$-conjugate $p^{\prime}$ must be nonnegative, since $f=p p^{\prime}$ is nonnegative. Hence $p$ is nonnegative with respect to every (real) place of $K$, and therefore $p$ is a sum of squares over $K$, being a quadratic form. Now Hillar's result [7] implies that $f$ is a sum of squares over $\mathbb{Q}$.

4.11. It remains to consider the case when $f=N_{K / \mathbb{Q}}(l)$ where $[K: \mathbb{Q}]=4$ and $l \in K[\mathrm{x}]$ is a linear form whose coefficients generate $K$. When $K / \mathbb{Q}$ has a quadratic subfield $L / \mathbb{Q}$, we can write $f=N_{L / \mathbb{Q}}\left(N_{K / L}(l)\right)$ and conclude that $f$ is a sum of squares over $\mathbb{Q}$, by the argument in 4.10 . So we can assume that $K / \mathbb{Q}$ has no proper intermediate field. This means that $\operatorname{Gal}(\overline{\mathbb{Q}} / \mathbb{Q})$ acts on $\operatorname{Hom}(K, \overline{\mathbb{Q}})$ as the alternating or symmetric group. Let $l_{i}=0(i=1,2,3,4)$ be the four Galois conjugates of the line $l=0$. When $l_{1}, \ldots, l_{4}$ fail to be in general position, all four meet in a common $\mathbb{Q}$-point. After a suitable coordinate change we are then in the case of binary forms, in which it is clear that $f$ is a sum of squares over $\mathbb{Q}$. The proof of Theorem 4.1 is complete. 


\section{Some Open QUESTIONS}

Here are several natural questions that arise in connection with the results of this paper. Let always $\mathrm{x}=\left(x_{0}, \ldots, x_{n}\right)$.

5.1. In Theorem 2.1 we constructed forms in $\mathbb{Q}[\mathrm{x}]$ that are sums of squares of forms over $\mathbb{R}$, but not over $\mathbb{Q}$. All our examples split over $\mathbb{C}$ as products of linear forms. Are there examples that are irreducible over $\mathbb{C}$ ? Are there examples that are strictly positive definite, i.e., that have no nontrivial real zeros? Are there examples that define a nonsingular projective hypersurface? (The last question is a common sharpening of the former two.)

5.2. Let $K$ be a real number field, and let $f$ be a form in $\mathbb{Q}[\mathrm{x}]$ that is a sum of squares of forms over $K$. When $K$ is totally real, it follows that $f$ is a sum of squares over $\mathbb{Q}$ (Hillar [7, c.f. also Section [1). Are there other sufficient conditions on $K$ that allow the same conclusion?

5.3. More specifically, let $K$ be a number field of odd degree, and assume that a form $f$ over $\mathbb{Q}$ is a sum of squares over $K$. Then, is $f$ a sum of squares over $\mathbb{Q}$ ?

5.4. We may generalize the last question to arbitrary linear matrix inequalities. Thus, let $A_{0}, \ldots, A_{r}$ be symmetric matrices of some size with rational coefficients, and assume that there exists $x=\left(x_{1}, \ldots, x_{r}\right) \in K^{r}$ such that the matrix $A(x):=$ $A_{0}+\sum_{i=1}^{r} x_{i} A_{i}$ is positive semidefinite with respect to every real place of $K$. If $[K: \mathbb{Q}]$ is odd, does there exist $x \in \mathbb{Q}^{r}$ such that $A(x)$ is positive semidefinite? For $r=1$, the answer is yes.

5.5. When $f \in \mathbb{Q}[\mathrm{x}]$ is any nonnegative form, there exists a sum of squares $h \neq 0$ of forms in $\mathbb{Q}[\mathrm{x}]$ such that $f h$ is a sum of squares of forms in $\mathbb{Q}[\mathrm{x}]$. Assuming that $f$ is a sum of squares of forms over $\mathbb{R}$, can we give an upper bound to $\operatorname{deg}(h)$, for example in terms of $n$ and $d=\operatorname{deg}(f)$ ?

Note that there is one case in which the results of this paper give an answer to this question, namely $(n, d)=(2,4)$. Here $\operatorname{deg}(h)=2$ suffices by 4.1 and 3.3 .

\section{REFERENCES}

[1] M. F. Anjos, J. B. Lasserre: Handbook on Semidefinite, Conic and Polynomial Optimization. Springer, 2012.

[2] V.I. Arnold, S. M. Gusein-Zade, A. N. Varchenko: Singularities of Differentiable Maps, Volume 1. Monographs in Mathematics, Birkhäuser, Boston, 1985.

[3] E. Artin: Über die Zerlegung definiter Funktionen in Quadrate. Abh. Math. Sem. Univ. Hamburg 5, 100-115 (1927).

[4] K. Belabas: Number field tables, generated by the Bordeaux computational number theory group (H. Cohen et al.) around 1995. Corrected version (2007). Available from pari.math.u-bordeaux1.fr/pub/pari/packages/nftables

[5] S. Boyd, L. Vandenberghe: Convex Optimization. Cambridge Univ. Press, Cambridge, 2004.

[6] D. Hilbert: Über die Darstellung definiter Formen als Summe von Formenquadraten. Math. Ann. 32, 342-350 (1888).

[7] Ch. Hillar: Sums of squares over totally real fields are rational sums of squares. Proc. Am. Math. Soc. 137, 921-930 (2009).

[8] E. L. Kaltofen, B. Li, Z. Yang, L. Zhi: Exact certification in global polynomial optimization via sums-of-squares of rational functions with rational coefficients. J. Symb. Comput. 47, $1-15$ (2012).

[9] J. B. Lasserre: Moments, Positive Polynomials and Their Applications. Imperial College Press, London, 2010. 
[10] A. Nemirovski: Advances in convex optimization: conic programming. Int. Cong. Math. vol. I, European Math. Soc., Zürich, 2007, pp. 413-444.

[11] R. Quarez: Tight bounds for rational sums of squares over totally real fields. Rend. Circ. Mat. Palermo 59, 377-388 (2010).

[12] M. Safey El Din, L. Zhi: Computing rational points in convex semialgebraic sets and sum of squares decompositions. SIAM J. Optim. 20, 2876-2889 (2010).

[13] W. Scharlau: Quadratic and Hermitian Forms. Grundl. math. Wiss. 270, Springer, Berlin, 1985.

[14] C. Scheiderer: Hilbert's theorem on positive ternary quartics: A refined analysis. J. Algebraic Geometry 19, 285-333 (2010).

[15] C. Scheiderer: In how many ways is a quartic a conic of conics? Data over $\mathbb{C}$ and over $\mathbb{R}$, available at www.math.uni-konstanz.de/ scheider/notes/TQ.details.pdf

[16] H. Wolkowicz, R. Saigal, L. Vandenberghe (eds.): Handbook of Semidefinite Programming. Theory, Algorithms, and Applications. Kluwer, Boston, 2000.

Fachbereich Mathematik and Statistik, Universität Konstanz, D-78457 Konstanz, Germany

E-mail address: claus.scheiderer@uni.constanz.de

$U R L:$ http://www. math. uni-konstanz/ ${ }^{\text {scheider }}$ 